\title{
Static Positional Nystagmus in the Healthy Vestibular System
}

DOI: $10.3766 /$ jaaa.18024

\author{
M. Dawn Nelson* \\ Larissa Mann* \\ Christine Nicholson* \\ Mark Lehman*
}

\begin{abstract}
Background: A repeat of the seminal 1973 study on static positional nystagmus (PN) using more accurate recording techniques.

Purpose: The purpose was to further characterize PN and, using current data, introduce new clinical criteria for its identification.

Research Design: Static PN was recorded in ten positions with vision denied. Each position was analyzed using age, gender, presence, direction, and persistence of nystagmus while taking into account the number of beats and mean slow-phase velocity (SPV).
\end{abstract}

Study Sample: One hundred healthy patients who were asymptomatic with no known neurological disorders were tested.

Intervention: No intervention was used.

Data Collection: Analysis of variance, descriptive statistics, and confidence intervals were used to describe results.

Results: Results showed $74 \%$ of normal participants had horizontal nystagmus in at least one position. Only $7 \%$ of the observed nystagmus was persistent. The average SPV was $2 \% \mathrm{sec}$. The mean number of positions in which nystagmus was observed was three. Neither age nor gender influenced the occurrence of nystagmus. Forty-three percent of the participants had vertical nystagmus in at least one position; however, the SPV was $2 \%$ sec or less.

Conclusions: The present study demonstrated that intermittent or persistent PN in four or fewer positions should not be considered pathological when the SPV is $4 \% \mathrm{sec}$ or less $(n=100)$. Observance of vertical nystagmus in one position should not be considered pathological if the SPV is $2 \%$ sec or less. Suggested positions for positional testing should include seated-upright, supine, head right, head left, head-hanging, and the precaloric ( $30^{\circ}$ supine) positions. Fixation when PN is observed is indicated.

Key Words: balance, electronystagmography, eye movements, nystagmus, positional nystagmus, static, vestibular, videonystagmography

Abbreviations: ANOVA = analysis of variance; BPPV = benign paroxysmal positional vertigo; CNS = central nervous system; ENG = electronystagmography; $\mathrm{HH}=$ head-hanging; $\mathrm{HHL}=$ head-hanging left; $\mathrm{HHR}=$ head-hanging right; $\mathrm{HL}=$ head left; $\mathrm{HR}=$ head right; $\mathrm{LL}=$ left lateral; $\mathrm{MRI}=$ magnetic resonance imaging; $\mathrm{PC}=$ precaloric; $\mathrm{PN}=$ positional nystagmus; $\mathrm{RL}=$ right lateral; $\mathrm{SPV}=$ slow-phase velocity; VNG $=$ videonystagmography

*Department of Communication Sciences and Disorders, Central Michigan University, Mount Pleasant, MI

Corresponding author: M. Dawn Nelson, Department of Communication Sciences and Disorders, Central Michigan University, Mount Pleasant, MI 48859; Email: nelso1md@cmich.edu

Larissa Mann is now at the Idaho Elks Hearing and Balance Center, Nampa, Idaho. Christine Nicholson is now at Children's Hospital of Philadelphia, Philadelphia, Pennsylvania.

This project was presented at the American Academy of Audiology Conference (AudiologyNOW!) in Dallas, TX, April 2009. 


\section{INTRODUCTION}

$\mathrm{N}$ ystagmus that is observed after a person's head or body is placed in a static position is known as positional nystagmus (PN). PN must be differentiated from positioning nystagmus, which is typically associated with benign paroxysmal positional vertigo (BPPV) and is the result of moving the body and head from one position to another. Differentiating positional from positioning nystagmus is sometimes challenging and the clinician must use careful patient history, results from other clinical tests, and knowledge of vestibular anatomy and disorders to aid in an accurate diagnosis (Appiani et al, 2001; Cakir et al, 2006).

The positional subtest of the videonystagmography (VNG) test battery identifies and records any eye movements resulting from a patient's head or head and body being placed in several static positions. These static positions can include but are not limited to supine and supine with only the head turned to the right or left and positions where both the head and body are turned to the right or left with eyes oriented straight ahead in the ocular orbit and vision denied. Patient eye movements are usually recorded for 20-30 sec after the head and body movements have ceased. Alerting tasks are implemented to prevent cognitive suppression of eye movements. (Nylén, 1953; Baloh, et al, 1987; Brandt, 1990; McGovern and Fitzgerald, 2008).

$\mathrm{PN}$ can occur in the horizontal plane for a given position or in the vertical plane for a given position. When horizontal, it is characterized as either right- or leftbeating nystagmus. When vertical, it is characterized as up- or down-beating nystagmus. Static PN can even change directions during a single static position (Bassani and Torre, 2011). It is believed to be caused from an imbalance in tonic activity within the vestibular labyrinths, the vestibular nerves or the central vestibular system leading to inappropriate activation of the vestibular ocular reflex and nystagmus. Horizontal PN has been associated with both peripheral as well as central vestibular disorders. Vertical PN, on the other hand, is usually indicative of a central nervous system (CNS) vestibular lesion (Bassani and Torre, 2011). A common cause of $\mathrm{PN}$ due to asymmetric eighth nerve and/or vestibular nuclear complex firing is vestibular neuritis (Lin et al, 1986). Other suggested causes of PN include structural and metabolic changes in the cupula that may arise with age or disease or differences in the specific gravity of the endolymph within the semicircular canals versus the cupula caused by alcohol ingestion. (Nylén, 1950; Jongkees, 1961; Baloh and Honrubia, 1990; Brandt, 1990; Glasauer et al, 2001). PN due to CNS dysfunction can be seen in patients with migraine, damage to the cerebellum or brainstem, cerebellum neoplasm resulting in compression of the 4 th ventricle, ponto-medullary disorders, other CNS disorders, or those with loss of blood supply to the basilar artery (Harrison and Ozsahinoglu, 1972; Watson et al, 1981; Bassani and Torre, 2011; Roberts, 2016).

Historically, the presence of PN was interpreted as a nonlocalizing finding, that is, the origin could be from peripheral or CNS damage that could not be determined. More recently, categorization of $\mathrm{PN}$ that is peripheral in nature has become more routine, especially in the presence of direction-fixed horizontal nystagmus with vision denied seen in several positions, including the seated-upright position (spontaneous nystagmus) and an accompanying significant unilateral weakness toward the slow phase of the PN (Barber and Wright, 1973; Lin et al, 1986; Shepard and Teilan, 1996).

Several authors recorded the presence of horizontal nystagmus during the positional subtests in persons who were medically normal, reporting no dizziness or imbalance. These studies used electrooculography or electronystagmography (ENG) recording techniques from electrodes placed around the outer canthi of each eye. They found that more than $80 \%$ of their healthy participants presented with nystagmus in at least one position (Barber and Wright, 1973; Mulch and Lewitzki, 1977; Coats, 1993; McAuley et al, 1996). Others have noted, however, that PN only occurs in the presence of peripheral or central vestibular pathology (Uemura et al, 1977; Rubin and Brookler, 1991).

Early studies on PN in normal participants might have been subject to the documented pitfalls of electrooculography/ENG that included the introduction of significant noise, electrical drift, and artifact. It is possible that these factors may have affected the accurate detection and analysis of the nystagmus. The more recent availability of computerized techniques that use infrared video goggles known as VNG has significantly facilitated the sensitivity, accuracy, quantification, and analysis of horizontal and vertical eye movements. VNG also allows the observation of torsional nystagmus, which enhances the diagnosis of BPPV (Waldorf et al, 1977; Kitamura et al, 1995; Vitte and Semont, 1995; Eckert and Gizzi, 1998; Jacobson and Shephard, 2008). For example, Lightfoot (2004) compared the calibration integrity of ENG and VNG systems and its impact on measuring the slow-phase velocity (SPV) of a nystagmus beat. A significant difference was observed between the initial and final calibration during ENG testing, suggesting that diagnoses may have been negatively influenced by faulty calibration. That is, results showed that SPV errors were $>20 \%$ for one third of the participants when calibration was not performed before each ENG subtest and that errors in corneo-retinal potential readings could lead to inaccurate SPVs and interpretation. Lightfoot (2004) suggested that at minimum, when using ENG, calibration be performed before each 
caloric irrigation. No such calibration errors were observed for VNG.

More recently, using VNG techniques, significant vertical and horizontal PN was observed in asymptomatic participants with "normal" vestibular function (Bisdorff et al, 2000; Geisler et al, 2000; Sunami et al, 2004; Jeffery, et al, 2017). The incidence of horizontal nystagmus ranged from $50 \%$ to $73 \%$ and $4.5 \%$ to $55 \%$ for vertical nystagmus. Unfortunately, little data were presented regarding directionality, SPV, or duration. Table 1 shows available data from studies on normal participants who demonstrated nystagmus during positional testing.

Despite these newer data, disagreement still exists regarding the occurrence of $\mathrm{PN}$ among healthy individuals without vestibular pathology (Roberts and Gans, 2008a,b).

Updated clinical insight regarding the incidence, duration, velocity, general description, and interpretation of PN using more accurate recording techniques with a large, medically normal participant pool seems warranted. Results should help answer many questions in this decade's long discussion. This study should help to further characterize static PN in healthy patients who are asymptomatic regarding vestibular symptoms. In addition, new clinical criteria for the identification of static PN will be presented.

\section{METHODS}

\section{Participants}

Twenty-seven males and 73 females $(n=100)$, ranging in age from 18 to 68 years, participated in this study. The participants were divided by age into three groups, ages 18 to $24(\mathrm{n}=53)$ years, 25 to 39 years $(\mathrm{n}=27)$, and 40 to 68 years $(\mathrm{n}=20)$. Otoscopy confirmed that ears were free from debris, redness, and visible perforation before testing. All participants had normal hearing and middle ear status confirmed by hearing screening, present acoustic reflexes elicited at normal sensation levels (re: pure-tone thresholds) and type A tympanograms characterized by peak middle ear pressure between +100 to $-150 \mathrm{daPa}$ and maximum relative compliance between 0.3 to $1.4 \mathrm{ml}$ (Margolis and Hunter, 1999). All participants had a negative history of dizziness, imbalance, or hearing loss and considered themselves in good health as determined by a written questionnaire. They were asked if they experienced feelings of unsteadiness, lightheadedness, or spinning. Participants were also queried about vestibular surgeries, ear infections, hearing loss, draining ears, and other neurological diagnoses. Participants were immediately excluded from the study if they answered yes to any of these symptoms or disorders. Alternate binaural bithermal water calorics were performed on each participant to ensure that the low-frequency vestibular ocular reflex response of the horizontal canals was confirmed as having normal function. The criteria used for normal caloric responses were $<25 \%$ unilateral weakness and $<30 \%$ directional preponderance (Henry, 1999; Baloh and Honrubia, 2001). Any volunteer who did not pass the selection criteria was excluded from the study. Ultimately, only one participant was eliminated from the study. Previous ear surgery was the disqualifying factor. No participants were excluded because of demonstration of a unilateral or bilateral caloric weakness. All participants were instructed to refrain from consuming alcohol and taking all but essential medications for 48 hours before testing.

Table 1. Previous Studies That Evaluated the Prevalence of PN in Participants without Vestibular Pathology

\begin{tabular}{|c|c|c|c|c|c|c|c|c|c|c|c|}
\hline & $\begin{array}{l}\text { Number of } \\
\text { Participants }\end{array}$ & $\begin{array}{c}\text { ENG } \\
\text { or VNG }\end{array}$ & $\begin{array}{l}\text { Number } \\
\text { of Test } \\
\text { Positions }\end{array}$ & $\begin{array}{c}\text { Vertical } \\
\text { Nystagmus }\end{array}$ & $\begin{array}{l}\text { Horizontal } \\
\text { Nystagmus }\end{array}$ & Persistent & Intermittent & Left-Beating & Right-Beating & $\begin{array}{l}\text { Mean } \\
\text { SPV }\end{array}$ & $\begin{array}{c}\text { Average } \\
\text { Number of } \\
\text { Positions } \\
\text { Nystagmus } \\
\text { Present }\end{array}$ \\
\hline Present study & 100 & VNG & 10 & $43 \%$ & $74 \%$ & $7 \%$ & $93 \%$ & $63 \%$ & $37 \%$ & 2 & 3 \\
\hline $\begin{array}{l}\text { Barber and } \\
\text { Wright (1973) }\end{array}$ & 112 & ENG & 8 & ---- & $83 \%$ & $34 \%$ & $66 \%$ & ---- & ---- & ---- & ---- \\
\hline Lin et al (1986) & 44 & ENG & 5 & ---- & $66 \%$ & ---- & ---- & ---- & ---- & ---- & ---- \\
\hline $\begin{array}{l}\text { McAuley } \\
\text { et al (1996) }\end{array}$ & 49 & ENG & 6 & --- & $88 \%$ & $6 \%$ & $82 \%$ & ---- & ---- & 2 & ---- \\
\hline Coats (1993) & 51 & ENG & 5 & ---- & $82 \%$ & ---- & ---- & Predominant & ---- & ---- & ---- \\
\hline $\begin{array}{l}\text { Geisler } \\
\text { et al (2000) }\end{array}$ & 29 & VNG & 11 & ---- & $55 \%$ & ---- & ---- & ---- & ---- & ---- & ---- \\
\hline $\begin{array}{l}\text { Bisdorff } \\
\text { et al (2000) }\end{array}$ & 40 & VNG & 2 & $55 \%$ & ---- & --- & ---- & ---- & ---- & ---- & ---- \\
\hline $\begin{array}{l}\text { Sunami } \\
\text { et al (2004) }\end{array}$ & 89 & VNG & 8 & $4.5 \%$ & $73 \%$ & ---- & ---- & ---- & ---- & ---- & ---- \\
\hline $\begin{array}{l}\text { Martens } \\
\text { et al (2016) }\end{array}$ & 75 & VNG & $\begin{array}{l}6 \text { Rotating } \\
\text { table }\end{array}$ & --- & $88 \%$ & ---- & ---- & ---- & ---- & 2.4 & ---- \\
\hline $\begin{array}{l}\text { Jeffery } \\
\text { et al (2017) }\end{array}$ & 90 & VNG & 4 & ---- & $50 \%$ & ---- & ---- & ---- & ---- & $<3$ & ---- \\
\hline
\end{tabular}




\section{Instrumentation}

The Grason-Stadler GSI 61 two-channel audiometer with E.A.R. Tone 3A insert earphones was used to complete the hearing screening. The Grason-Stadler GSI TympStar Middle Ear Analyzer was used to confirm normal middle ear status. Video-oculographic (VNG) recordings of eye movements were obtained and analyzed using the 2-channel, GN Otometrics ICS Medical Chartr VNG system, model number NCI-480, and the ICS Medical VG-30 goggles (GN Otometrics, Schaumburg, IL). Darkpupil tracking technology was used to record eye movements. This technique used off-axis infrared illumination, from goggle-mounted cameras directed over each eye, to generate contrast between the iris and the pupil. The exact pupil position was detected and measured using a detection algorithm, or Hough transform (Jacobson and Shephard, 2008). Cameras were secured to the goggles relative to the head to make certain that any recorded eye movement was not mistaken for head and eye movement (Clarke et al, 2002; Jacobson, and Shephard, 2008). Water calorics were conducted using the ICS water caloric system.

\section{Procedures}

All positional and caloric testing was conducted in a dark room. Each participant was seated on an examination table and fit with the dark-pupil tracking video goggles. To prevent visual fixation, a light proof cover was placed over the front of the goggles. Although vision was denied, participants were directed to keep their eyes open throughout the testing period. For each participant, calibration was accomplished by having participants track a sinusoidal target moving $\pm 20^{\circ}$ laterally and then vertically from center gaze. Participants performed mental alerting exercises during testing (Davis and Mann, 1987; McGovern and Fitzgerald, 2008). For example, they were asked to list items found in a kitchen or to list holidays.

During positional testing, each participant was placed into ten different static head or head/body positions for a duration of $100 \mathrm{sec}$ each. Eye movements were recorded for the entire $100 \mathrm{sec}$ for each position. Individual positions included seated-upright, supine, supine with head right (HR), supine with head left (HL), left lateral (LL), right lateral $(\mathrm{RL})$, head-hanging $(\mathrm{HH})$, head-hanging right (HHR), head-hanging left (HHL), and precaloric (PC) that is defined as supine with the head tilted upward at a $30^{\circ}$ angle. The order of the ten positions was randomized for each participant.

Following positional testing, bilateral bithermal water caloric testing was conducted. Water temperature was $44^{\circ} \mathrm{C}$ for warm irrigations and $30^{\circ} \mathrm{C}$ for cool irrigations. Warm irrigations were conducted first followed by cool irrigations (ANSI, 2009).

\section{Criteria for the Identification and Categorization of Nystagmus}

To prepare for data analysis, nystagmus was identified, counted, and measured. Nystagmus for a given position was deemed present when three consecutively occurring beats were identified during the 100 -sec recording epoch. Each nystagmus beat had to have an obvious fast and slow-phase component with an SPV that was greater than or equal to one degree per second $(1 \% \mathrm{sec})$ to be considered for analysis. Nystagmus that contained square wave components was not included in the analysis (Herishanu and Sharpe, 1981; Leigh and Zee, 1999). Nystagmus was considered persistent if it occurred for at least $80 \mathrm{sec}$ or $80 \%$ of the $100 \mathrm{sec}$ time window, otherwise it was considered to be intermittent. For each position, the 100-sec tracing was divided into ten blocks of 10 sec recordings each. Individual nystagmus beats for each time block were counted and the SPV of each beat was measured. If nystagmus was present for a given position, it was categorized as direction-fixed or direction-changing. Horizontal nystagmus was considered direction-fixed if it was only right-beating or only left-beating across all positions. Horizontal nystagmus was considered direction-changing if the participant had both right-beating and left-beating nystagmus across different positions. Direction-changing in a single position was defined as right-beating and left-beating nystagmus recorded for one position within the $100 \mathrm{sec}$ epoch.

\section{Data Analysis}

Analysis of variance (ANOVA) and descriptive statistics, including the mean, frequency, and 95th percentiles were used to analyze the data.

\section{RESULTS}

$2 \times 3 \times 10$ ANOVA was performed to determine if
there were significant main effects for age or gender on SPV across positions. Results showed no significant main effect for age or gender [age: $F(2,94)=0.333$, $p=0.718$; gender: $F(1,94)=1.908, p=0.17]$. Therefore, participants were considered as one group for all subsequent analysis. There was a significant main effect for SPV across positions $[F(9,846)=2.766, p=0.003]$ as well as a significant main effect for positions $[F(9,846)=$ 2.691, $p=0.004]$. Pair-wise comparisons revealed that the only significant difference between positions was found between the spontaneous and HL positions.

Horizontal PN was present for $74 \%$ of the participants in at least one position and the remaining $26 \%$ of the participants had no nystagmus for any position $(\mathrm{N}=100)$. No participant experienced feelings of imbalance or dizziness before, during, or after testing. As shown in Figure 1, PN was most prevalent in the HL 


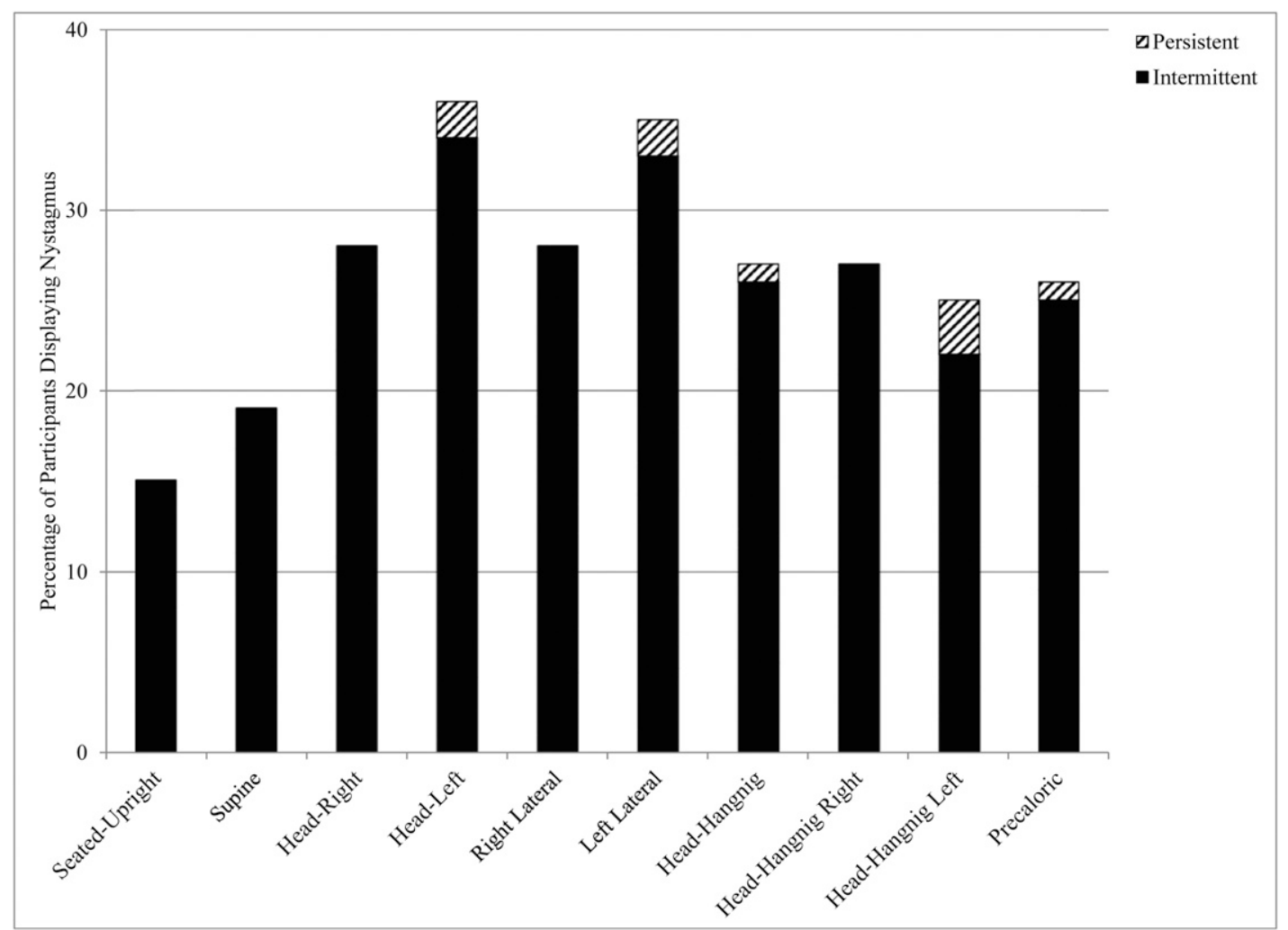

Figure 1. Percent of nystagmus beats, both intermittent and persistent, for each of the ten test positions. PN was most prevalent in the HL position.

position. Thirty-seven percent of all observed nystagmus occurred in this position. There was no statistical difference between the nystagmus in the LL position and the HL position $[T(99)=208, p=0.836]$. Furthermore, there was no statistical difference between the nystagmus in the RL position and the HR position $[T(99)=0, p=1.0]$. The occurrence of spontaneous nystagmus in the seated-upright position was the least prevalent. Only $15 \%$ of the time was nystagmus observed during this position. For each position tested $(\mathrm{N}=10)$, nystagmus was recorded in $>15 \%$ of the participant pool. For no single position was nystagmus recorded in $>36 \%$ of the participants. The mean number of positions in which horizontal nystagmus was present was three positions. Figure 2 demonstrates the percent of participants who displayed nystagmus by number of positions. Eleven percent of the participants had nystagmus in three positions. Eighteen percent had nystagmus in one position and one normal participant had nystagmus in nine of the ten positions.

A $2 \times 3 \times 10$ ANOVA was performed to see if there was a significant effect for age or gender on duration, that is, intermittence and persistence of nystagmus across positions. Results showed no significant main effect for age $[F(2,94)=0.455, p=0.636]$. However, a significant main effect was shown for gender and duration $[F(1,94)=4.277, p=0.041$; duration $F(9,846)=$
$2.691, p=0.004]$. Including only the $74 \%$ of the participants with horizontal PN, 7\% of them had persistent nystagmus in at least one position and $93 \%$ of the participants had intermittent nystagmus for at least one position. Figure 1 graphically represents the occurrence of intermittent versus persistent nystagmus across the ten positions.

For all observed nystagmus, that is, 869 nystagmus beats, the mean SPV was $2 \%$ sec, the minimum SPV was $1 \%$ sec, and the maximum SPV was $9 \%$ sec. The 95 th percentile for SPV was $4 \%$ sec, that is, $95 \%$ of the recorded nystagmus was $4 \%$ sec or less. Only $5 \%$ of the participants had nystagmus with an SPV greater than $4 \%$ sec.

Left-beating nystagmus was more prevalent than right-beating nystagmus. Table 1 shows that $63 \%$ of all nystagmus beats recorded were left-beating. Right-beating nystagmus only occurred $37 \%$ of the time.

Of patients demonstrating $\mathrm{PN}(\mathrm{N}=74)$, directionfixed nystagmus was present in $76 \%$ of the participants and the remaining $24 \%$ of the participants had direction-changing nystagmus. Of those with directionchanging nystagmus, $11 \%$ had ageotropic nystagmus for each position where nystagmus was present and $61 \%$ had geotropic nystagmus. The remaining $28 \%$ of the participants had a mixture of ageotropic and geotropic nystagmus (Jungmoo et al, 2009). No participant 


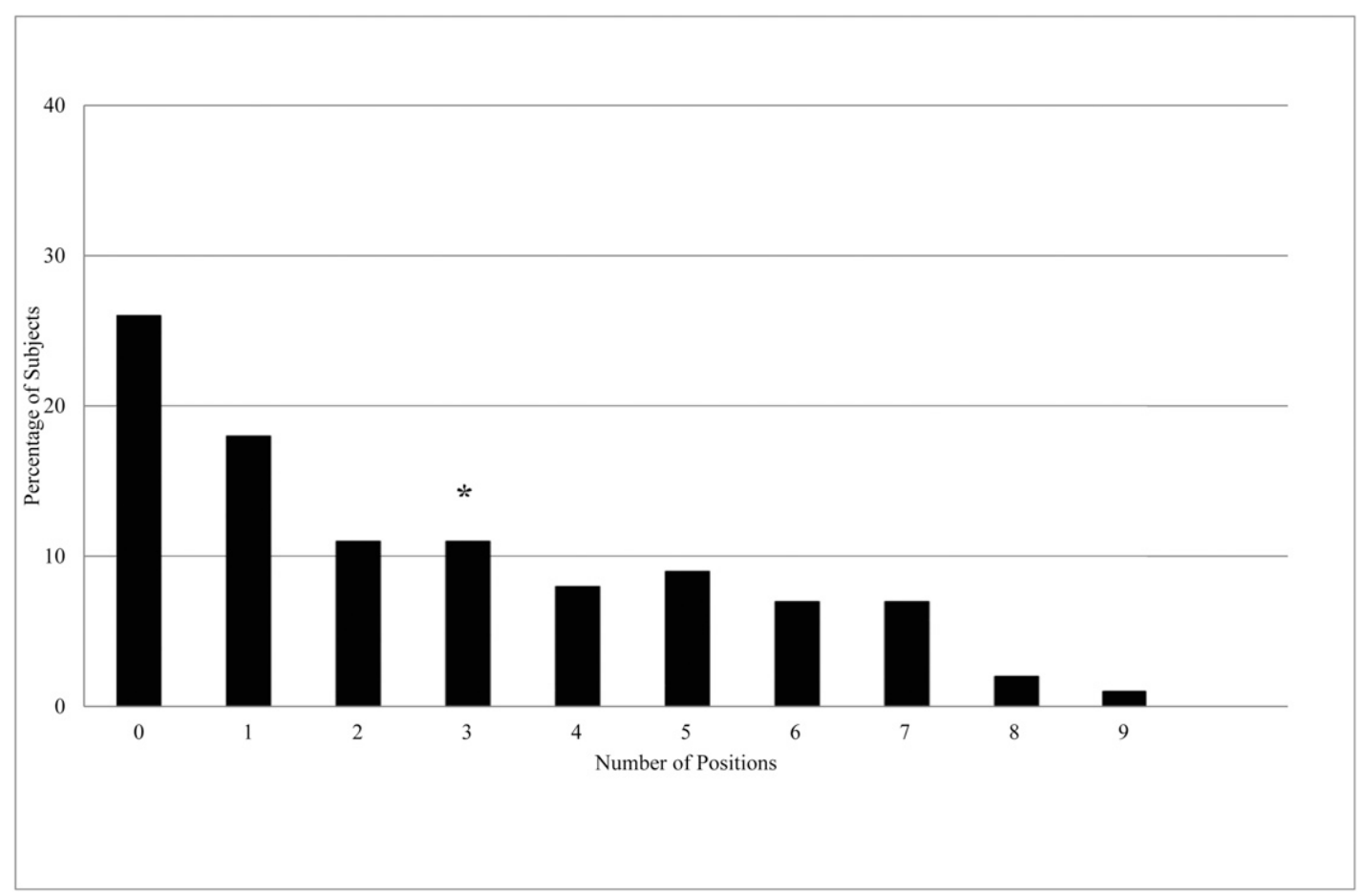

Figure 2. The mean number of positions in which horizontal nystagmus was present was three positions (11\%).

demonstrated torsional nystagmus or direction-changing nystagmus in a single position.

Forty-three percent of the participants had vertical nystagmus in at least one position. The remaining $57 \%$ had no recorded or observed vertical nystagmus in any position. The average SPV for vertical nystagmus was $1 \%$ sec. On average, vertical nystagmus was present in only one position.

\section{Clinical Criteria for Pathological Nystagmus}

Table 2 reviews two commonly used sets of clinical criteria that can be applied to recorded positional criteria and two newly suggested sets (Barber and Stockwell, 1980; Shepard and Telian, 1996). The number of healthy participants $(\mathrm{N}=100)$ who met the criteria for suspected pathologic nystagmus are also presented in Table 2. Separate calculations are provided for ten test positions, eight test positions, and six test positions. The ten test positions included sitting upright, supine, HR, HL, HH, PC ( $30^{\circ}$ supine), RL, LL, HHR, and HHL. When the criteria are analyzed with eight positions, HHR and HHL are excluded because PN in these positions can be evaluated during the Dix-Hallpike test. The criteria are then analyzed with six test positions. Here, HHR and HHL are excluded as well as the RL and LL positions because lateral positions can be included in a test battery if neck involvement is in question. Table 2 shows that the number of participants who met the criteria for significant PN was highly dependent on the number of positions tested, that is, as the number of positions tested decreased, fewer participants met the criteria for suspected pathology.

Using Barber and Stockwell's (1980) criteria, the total number of "flagged" participants decreased from 32 participants to 13 as the number of positions tested decreased from 10 positions, then to 8 and eventually to 6 . Shepard and Teilan's (1996) more liberal criteria showed the same trend. Participants meeting their criteria decreased from eight to three as the number of positions tested decreased. Suggested criteria \# 1 from this study decreased from 22 to 5 participants identified as suspected pathology.

Table 2 also illustrates that the number of "flagged" participants (participants meeting the criteria for significant static $\mathrm{PN}$ ) was influenced by the degree to which intermittent nystagmus was considered. That is, the overall total of "flagged" participants was greatly influenced by the number of positions, demonstrating intermittent $\mathrm{PN}$, required to meet the criteria of significance. Table 2 shows that when ten positions were considered for the analysis with a criterion for significance (pathological) set at "intermittent nystagmus in at least 8 positions," only 3 participants qualified; "intermittent in at least 6 positions," 15 qualified; and "4 positions," 31 qualified. Shepard and Teilan (1996) used eight positions. For suggested criteria \#1 and \#2, the number of positions needed with intermittent nystagmus was varied between six and four positions because these numbers are closer to the average number of four positions in which PN was observed. 
Table 2. Criteria for Significant PN

\begin{tabular}{|c|c|c|c|}
\hline Number of Participants Meeting Criteria & 10 pos & 8 pos & 6 pos \\
\hline \multicolumn{4}{|l|}{ Barber and Stockwell, 1980 (adapted from Barber and Wright, 1973) } \\
\hline 1. Direction-changing in a single position. & 0 & 0 & 0 \\
\hline 2. SPV $>6 \%$ sec for the three strongest consecutive beats in a single position. & 0 & 0 & 0 \\
\hline 3. Persistent in three positions. & 1 & 1 & 0 \\
\hline 4. Intermittent in four positions. & 31 & 23 & 13 \\
\hline Total no. of participants meeting criteria & 32 & 24 & 13 \\
\hline \multicolumn{4}{|l|}{ Shepard and Telian (1996) } \\
\hline 1. Direction-changing in a single position. & 0 & 0 & 0 \\
\hline 2. SPV $>5 \%$ sec in a single position. & 5 & 5 & 3 \\
\hline 3. SPV $<6 \%$ sec-persistent nystagmus in four or more of $8-11$ positions. & 0 & 0 & 0 \\
\hline 4. SPV $<6 \%$ sec-intermittent in all (of 8 ) positions. & 3 & 0 & 0 \\
\hline Total no. of participants meeting criteria & 8 & 5 & 3 \\
\hline \multicolumn{4}{|l|}{ Suggested criteria \#1 (2018) (fewer referrals) } \\
\hline 1. Direction-changing in a single position. & 0 & 0 & 0 \\
\hline 2. SPV $>5 \%$ sec for the three strongest consecutive beats in a single position. & 5 & 5 & 3 \\
\hline 3. SPV $<6 \%$ sec-persistent nystagmus in three or more positions. & 1 & 1 & 0 \\
\hline 4. SPV $<6 \%$ sec-intermittent in at least six positions. & 15 & 7 & 0 \\
\hline 5. Combination of persistent and intermittent nystagmus in four positions. & 1 & 3 & 2 \\
\hline Total no. of participants meeting criteria & 22 & 16 & 5 \\
\hline \multicolumn{4}{|l|}{ Suggested criteria \#2 (2018) (more referrals) } \\
\hline 1. Direction-changing in a single position. & 0 & 0 & 0 \\
\hline 2. SPV $>5 \%$ sec for the three strongest consecutive beats in a single position. & 5 & 5 & 3 \\
\hline 3. SPV $<6 \%$ sec-persistent nystagmus in four or more positions. & 0 & 0 & 0 \\
\hline 4. SPV $<6 \%$ sec-intermittent in at least four positions. & 31 & 23 & 13 \\
\hline 5. Combination of persistent and intermittent nystagmus in four positions. & 1 & 3 & 2 \\
\hline Total no. of participants meeting criteria & 37 & 31 & 18 \\
\hline
\end{tabular}

\section{DISCUSSION}

$\mathrm{P}$ ositional nystagmus results from the head or body being slowly placed into a static position and should not be confused with positioning nystagmus, which is associated with specific movements used to diagnose BPPV. The present data suggest that the mere observance of PN during a vestibular evaluation may not indicate a lesioned vestibular system because $74 \%$ of the participants $(\mathrm{N}=100)$ demonstrated horizontal $\mathrm{PN}$ in at least one position and that for each position tested, horizontal nystagmus was recorded in more than $15 \%$ of the participant pool. Left-beating nystagmus occurred much more of the time ( $63 \%$ of the time) than right-beating nystagmus. It might be said that there was a left-beating dominance for nystagmus direction. Results also indicated that horizontal $\mathrm{PN}$ in three or four positions, when the SPV is $4 \%$ sec or less, may be a normal variant as well as the observance of vertical nystagmus in just one position if the SPV is $2 \%$ sec or less. Reports to the contrary, that PN is always pathological (Uemura et al, 1977; Rubin and Brookler, 1991; Roberts and Gans, 2008a,b), were not confirmed.

\section{Comparison with Other Studies}

Data from the present study are similar to results from previous studies that examined the incidence of
PN in normal participants. These data are reviewed in Table 1 (Barber and Wright, 1973; Lin et al, 1986; Coats, 1993; McAuley et al, 1996; Geisler et al, 2000; Sunami et al, 2004; Marten et al, 2016; Jeffery et al, 2017). The incidence of horizontal nystagmus in healthy participants is fairly consistent (50-88\%) across most studies (66-88\%), although at least two report incidence rates of 50-55\% (Geisler et al, 2000; Jeffery et al, 2017). This consistency holds even though the method of recording nystagmus, the number of participants tested, and the number of positions tested differed. The mean SPV, approximately $2-3 \%$ sec, seen among these studies, also remains consistent. For example, McAuley et al (1996) used ENG techniques to evaluate PN in six positions in 49 participants. They reported that the incidence of horizontal nystagmus was $88 \%$, the mean SPV was $2 \%$ sec, and the incidence of persistent and intermittent nystagmus was $6 \%$ and $82 \%$, respectively. Details further describing the nystagmus such as the incidence of vertical nystagmus or the mean numbers of positions where nystagmus was observed were not available.

Martens et al (2016) used a motorized rotating tilt table to position the entire body of their normal volunteers $(\mathrm{N}=75)$ into six test positions. Patients were tested in the seated-upright, supine, RL, LL, HHR, and HHL positions. In the $\mathrm{HH}$ positions, the participant was tilted 
such that the feet (and lower body) were elevated superior to the rest of the body. The reported incidence of PN was $88 \%$ with a mean SPV of $2.4 \%$ sec. They combined, however, horizontal, vertical, and oblique nystagmus in their analyses. It should be noted that $55 \%$ of their reported nystagmus was seen in the HH/lower body elevated positions. Figure 1 shows that this was not the case in the present study. A greater influence of gravity in these positions and its impact on the vestibular organ inside the ampulla, in their (Martens et al, 2016) participants, seems possible.

More recently, Jeffery et al (2017) compared PN across four positions in 90 control participants from Rogers et al (2014) with 90 of their balance patients. Positions tested were supine, supine with the head tilted $30^{\circ}$ (PC position), RL, and LL. They reported that Rogers et al (2014) found horizontal nystagmus in 50\% of the control participants. Furthermore, they reported that the mean SPV was less than $3 \%$ sec. They defined the maximum 95th CI for SPV across positions as $2.3^{\circ} / \mathrm{sec}$. They suggested a "threshold" of $3^{\circ}$ be used to determine a normal average SPV for horizontal nystagmus and persistent nystagmus in four positions. These data seem to be consistent with the present findings regarding incidence and mean SPV in a normal population. Differences in incidence could be related to number of positions used. While they did not report the incidence of vertical nystagmus from the control group, they suggested, based on their patient data, that vertical upbeat nystagmus in any position that is $7 \%$ sec or greater be considered pathological and needed further investigation. For their patient data $(\mathrm{N}=90)$, Jeffery et al (2017) found that $75.6 \%$ of the participants demonstrated nystagmus in at least one position. Like Martens et al (2016), they combined horizontal, vertical, and oblique nystagmus in their calculations making direct comparisons regarding the presence of geotropic, ageotropic, or direction-fixed nystagmus difficult. In addition, they used a criterion of "PN in two or more positions" for inclusion in those analyses and included data from other studies. Jeffery et al (2017) did not provide definitive diagnoses or confirmation of a central or peripheral vestibular lesion in their balance patients, so correlations between the patient data and their PN or calculations of sensitivity and specificity could not be made. In their opinion, any nystagmus exceeding $3 \%$ sec for any position or that is persistent and/or vertical (exceeding $7 \%$ sec) is considered probable pathologic.

Interestingly, there are some important differences between our data and the seminal report on the participants by Barber and Wright (1973). Our mean SPV of $2 \%$ sec is much lower than the $9 \%$ sec guidelines for pathological nystagmus suggested by Barber and Wright (1973) or the $6 \%$ sec suggested by Barber and Stockwell (1980). The 95 th percentile for SPV of $4 \%$ sec and the maximum SPV of $9 \%$ sec in the present study were lower than Barber and Wright's (1973) 95th percentile for maximum average SPV of $9 \% \mathrm{sec}$ and the maximum SPV of $12 \%$ sec. It is very likely that technical issues such as the strip-chart recorder combined with ENG techniques used by Barber and Wright (1973) versus computerized VNG techniques used in the present study account for some of the differences. Strip-chart ENG recordings can compromise the sensitivity and accuracy of nystagmus identification. Also, they can introduce significant noise, electrical drift, and artifact to recordings, which can affect the quantification and analysis of horizontal as well as vertical eye movements (Waldorf et al, 1977; Kitamura et al, 1995; Eckert and Gizzi, 1998).

\section{Test Positions}

As shown in Figure 1, the incidence of PN in the HL position and the LL positions was similar. This similarity between positions was also seen between the HR and RL positions. Consideration should be given to the elimination of the RL and LL positions from the VNG test battery, especially if a cervical vertigo screening test is negative (Clendaniel and Landel, 2007; Roberts, 2016). Perhaps, the lateral positions should be substituted for HR and HL if the patient complains of neck pain or stiffness during the case history portion of the session, the patient is unable to rotate the neck, if they fail the cervico-vertigo screening, or if the observed PN from neck rotation is in question. Our data show that only nine participants had static PN in only the LL or only the RL positions and not also in the HL, HR, or opposite-lateral positions. That is, only nine participants would have been missed by eliminating the lateral positions. These nine participants could possibly be identified as pathologic through careful case history, failure to fixate in other test positions, or through other clinical vestibular tests that help differentiate peripheral from central such as the caloric test (Maire and Duvoisin, 1999; Roberts, 2016). Also, it may be possible to eliminate the HHR and HHL positions from the protocol because the presence of PN in these positions can be assessed during the Dix-Hallpike test. Based on the present evidence, suggested positions for positional testing, without vision, might include sitting upright otherwise known as test of spontaneous nystagmus, supine, $\mathrm{HR}, \mathrm{HL}, \mathrm{HH}$, and the $\mathrm{PC}\left(30^{\circ}\right.$ supine) positions.

\section{Direction of Nystagmus}

Seventeen of the 100 healthy participants in the present study or $23 \%$ of only those showing static PN $(\mathrm{N}=17 / 74)$ had direction-changing nystagmus. Lin et al (1986) found that when their normal participants demonstrated static $\mathrm{PN}$, it was direction-changing nystagmus only $13.8 \%$ (4/29) of the time, slightly lower. They 
also calculated from chart review the overall incidence of direction-changing nystagmus for all of their 1,196 balance patients and 44 control participants, not just those with PN. Forty-five percent or $3.8 \%$ of their total patient population $(\mathrm{N}=1,196)$ and $9 \%(\mathrm{~N}=4 / 44)$ of their total healthy participants showed directionchanging nystagmus. A careful comparison of the two studies shows that the methods for carrying out the studies, methods of testing, number of different testers, the number of positions tested, the criteria for determining presence of nystagmus, and length recording epoch may have contributed to the differences between the studies. Incidentally, Lin et al (1986) reported that $76 \%$ of their patient participants $(\mathrm{N}=1,196)$ had PN; however, they failed to separate horizontal from vertical or oblique nystagmus. Direct comparisons are difficult. They did conclude, however, that the presence of direction-changing nystagmus does not necessarily indicate disease or localize the site of lesion. Specifically, Lin et al (1986) found that for $48 \%$ of their balance patients, they could find no definite diagnosis (patients with direction-changing nystagmus). Twenty-two percent of these patients had a confirmed central vestibular disorder such as cerebellar degeneration, brainstem infarction, multiple sclerosis, or other neurological disorder, and $30 \%$ percent had a peripheral vestibular lesion such as Meniere's disease, unilateral hypofunction of unknown etiology, or an acoustic neuroma.

Maire and Duvoisin (1999) looked at a group of 43 patients with either direction-fixed $(\mathrm{N}=35)$ or directionchanging $(\mathrm{N}=8) \mathrm{PN}$. They concluded that site of lesion, that is, peripheral versus central vestibular lesions could be determined in all of their patients by performing the failure of fixation suppression test when PN was observed. For example, eight of their patients with direction-fixed nystagmus were incapable of suppressing their PN. Each of these patients was found to have lesions in the posterior fossa. Pathology included tumors that invaded either the cerebellum or the pons. Each of their participants (18.8\%) with direction-changing nystagmus was able to suppress their nystagmus when they fixated. They noted that two of their patients who were able to fixate eventually were diagnosed with brainstem tumors, although they showed no clinical signs of a central lesion, and only the presence of PN. The addition of the failure of fixation suppression test when PN was seen significantly improved the clinician's ability to localize the lesion.

\section{Length of Recording}

Like McAuley et al (1996), no difference was found across time for number of nystagmus beats and SPV. The number of beats as well as the SPV did increase 20 to $30 \mathrm{sec}$ into the recording but decreased almost to the initial low level during the 60 to $70 \mathrm{sec}$ after recording onset. Low-level nystagmus activity during the first 30 sec of the recording may indicate that nystagmus was not caused by moving the patient into different positions.

\section{Criteria to Determine Significant PN}

Traditionally, clinical criteria have been applied to recorded nystagmus obtained during the positional subtest of the VNG or ENG test battery. The intent is to determine if the $\mathrm{PN}$ is nonpathologic or pathological. Patients with pathological $\mathrm{PN}$ may require further evaluation, monitoring, or treatment. Applying clinical criteria to positional results seems reasonable to avoid over referral of patients for expensive follow-up testing (Barber and Wright, 1973; Barber and Stockwell, 1980; Shepard and Teilan, 1996). Table 2 presents several clinical criteria or guidelines to analyze and consider.

All guidelines assume positional testing without vision and do not address interpretation of results when a patient has PN with vision. Data from these 100 healthy participants showed that $95 \%$ of the participants demonstrated an average SPV of $4 \%$ sec or less, in fact, the mean SPV was only $2 \%$ sec. If the exact mean SPV of $2.1(1 \mathrm{SD}=1.3)$ from our data is added to two standard deviations $(4.6 \% \mathrm{sec})$, then any nystagmus seen that is $5 \%$ sec or less could be considered typical. Lin et al (1986) only studied direction-changing nystagmus in a clinical population and summarized that all nystagmus in their group of participants had an SPV greater than $4 \%$ sec. The Barber and Stockwell (1980) guidelines used $>6 \%$ sec of the three strongest consecutive beats in a single position as their cutoff for suspected pathology. In their guidelines, Shepard and Teilan (1996) lowered this cutoff to $>5 \%$ sec, and this value was maintained in the suggested criteria. Five participants met the criteria with this cutoff of $>5 \%$ sec, and none met the criteria with a cutoff of $>6 \%$ sec. Said differently, there was a $5 \%$ false-positive rate using ten and eight positions and a $3 \%$ false-positive rate for six positions when using $>5 \%$ sec as the cutoff instead of using $>6 \% \mathrm{sec}$ which gave a false-positive rate of $0 \%$. Because no gold standard such as magnetic resonance imaging (MRI) was used on the participants in this present study the false-negative rate could not be calculated. Using a false-positive rate of $5 \%$ and an estimated cost of an MRI to be between 500 and 4,000 dollars, it can be estimated that between $\$ 2,500.00$ and $\$ 20,000.00$ may be spent on further testing of these five participants. It may be possible to further lower this false-positive rate by having the participant fixate in any given position where static PN is observed (Maire and Duvoisin, 1999; Roberts, 2016).

Differences in the definition of persistent nystagmus may account for the 5\% incidence of persistent nystagmus in the present study and the $34 \%$ reported by Barber and Wright (1973). Barber and Wright (1973) used a 
cutoff of $35 \%$ of the tracing, whereas nystagmus had to be present for $80 \%$ of the tracing or $80 \mathrm{sec}$ for it to qualify for the present analysis. The incidence of intermittent nystagmus was more similar among the three studies. These data were not available for the other studies.

As stated previously, the method of testing, that is, ENG versus VNG, the number of test positions used, and the number of test positions required when evaluating intermittent nystagmus may have had combined influence on the number of participants meeting the criteria for significant PN. Barber and Stockwell (1980) using ENG identified 31 participants as having significant static PN when testing ten test positions and a level of significance of "intermittent in four positions," whereas only 15 participants were identified using suggested criteria \#1 which used ten test positions, VNG, and a clinical criteria of "intermittent nystagmus in six positions." Also, the number of participants meeting the level of significance for positional static nystagmus dropped to zero from 15 (using suggested criteria \#1) when the number of positions was changed from 10 to 6 .

For the suggested sets of criteria, a fifth guideline, a combination of persistent and intermittent nystagmus in four positions, was included. Depending on the number of test positions used, between one and three of the 100 participants met this criterion. Again, calculating a fixation index to observed PN might lower this number (Maire and Duvoisin, 1999; Bertholon et al, 2002; Roberts, 2016).

The obvious absence of a set of criteria from Barber and Wright (1973) is deliberate. Careful review of their suggested criteria reveals that the initial guidelines were significantly revised, reported, and printed in the book by Barber and Stockwell (1980). Among other recommendations, the 1973 guidelines state that pathologic PN is suspected if there is: (a) a single beat of nystagmus with an SPV of $14^{\circ} / \mathrm{sec}$ or greater; (b) spontaneous nystagmus; (c) a maximum average SPV of $9 \%$ sec or greater for a burst (three beats) of nystagmus; (d) persistent or intermittent nystagmus in six or more positions and; (e) a combination of persistent and intermittent nystagmus in eight positions. They further stated that no $>4$ or 5 positions may be needed for the battery even though their criterion was based on 8 positions.

Overall, the present data show that when suggested criteria \#1, using only six positions is applied there is only a $5 \%$ false-positive rate. Only five of the 100 asymptomatic, otherwise healthy participants would have been identified as having significant static PN. Given the previous discussions on the elimination of certain positions, the SPV cutoff point and the influence of intermittent and persistent nystagmus, it seems reasonable to assume that this criteria might prove clinically efficient, yielding a very reasonable false-positive rate in a balance clinic. Suggested criteria \#2 is a bit more liberal when it comes to persistent nystagmus and more conservative when it comes to intermittent nystagmus. It yielded a false-positive rate of $18 \%$ when six positions were considered.

Suggested criteria \#1 seems to be a great compromise and is the preferred choice by our group. In retrospect, the addition of the fixation suppression test in a given position, if nystagmus was observed, may have shown even more promising results. Fixating has been shown to add to the diagnostic value of the positional subtests better categorizing patients into either central or peripheral vestibular lesion groups and minimizing the number of patients previously labeled with a nonlocalizing vestibular lesion (Maire and Duvoisin, 1999). Table 3 outlines an evidence-based protocol for PN that includes the preferred criteria, fixation, and other suggestions for the balance clinician. The addition of imaging for patients who cannot suppress their PN would add to its sensitivity (Lin et al, 1986; Maire and Duvoisin, 1999; Nam et al, 2009). The Appendix displays this information in a flowchart.

Table 3. An evidence-based criteria and protocol for the static positional subtest of the VNG test battery. The protocol assumes the positions, seated upright, supine, head-right, head-left, head hanging and supine with the head lifted $30^{\circ}$ (precaloric condition). The predictive value for this criteria was $95 \%$ in subjects where a healthy vestibular system was assumed.

Patients should meet one or more of the following six criteria for significant PN

1. Direction-changing in a single position.

2. SPV $>5 \%$ sec for the three strongest consecutive beats in a single position.

3. SPV $<6 \%$ sec-persistent nystagmus in three or more positions.

4. SPV $<6 \%$ sec-intermittent in at least six positions.

5. Combination of persistent and intermittent nystagmus in four positions.

6. Vertical nystagmus in a single position if the SPV $>2 \%$ sec or if present in more than one position.

In addition, clinicians should:

Check for suppression of PN for each position where PN is observed to help localize the lesion as either central or peripheral.

Perform a careful case history and cervico-vertigo screening to rule out neck problems. If neck problems exist, substitute or include the lateral positions.

Conduct the Dix Hallpike and the Roll tests prior to static positional testing to rule out BPPV.

Interpret PN results as part of a test battery along with other vestibular test results.

Always categorize PN as either direction-fixed or directionchanging. 


\section{Limitations}

The strength of our findings would improve if we had been able to administer other clinical techniques in our experimental test regimen. The video head impulse tests would have documented the absence of a high frequency vestibular lesion in the horizontal or vertical semicircular canals. The ocular vestibular-evoked myogenic test would have confirmed normal function of the utricle and/or superior branch of the 8th cranial nerve, although the caloric test probably could be seen as a cross check for normal 8th cranial nerve function. Jeffery et al (2017) did the cervical vestibular-evoked myogenic test on their normal participants and found no correlation between those results and static $\mathrm{PN}$; however, it could have been conducted in this study to rule out any saccular lesions. Another limitation of this study is that the incidence of migraines was not specifically tallied, although participants were queried about ailments, diagnoses, and neurological disorders.

\section{Summary}

This study demonstrated that PN can be observed in healthy people and that labels of pathologic nystagmus should be given with caution following an evidencebased criterion. Also, the clinician should always use clinical judgment, including case history and other clinical signs, to properly diagnose pathologic patients that fall outside the strict guidelines and are thereby missed. The suggested criteria \#1 had a predictive value of $95 \%$ for normal participants and should be considered in the future. Perhaps a large follow-up study using these criteria that compares participants with significant PN with healthy participants would provide useful insight into not-yet-detected vestibular lesions. This study should separately evaluate horizontal, vertical, and oblique nystagmus; analyze several aspects of the nystagmus; test the criteria against a gold standard such as MRI and/or cerebral angiography; and use the fixation index when nystagmus is observed (Bertholon et al, 2002; Choi et al, 2013).

\section{REFERENCES}

American National Standards Institute (ANSI). (2009) Procedures for Testing Basic Vestibular Function. (ANSI/ASA S3.45-2009 [Revision of ANSI S3.45-1999]). New York, NY: ANSI.

Appiani G, Catania G, Gagliardi M. (2001) A liberatory maneuver for the treatment of horizontal canal paroxysmal positional vertigo. Otol Neurotol 22:66-69.

Baloh RW, Honrubia V. (1990) Clinical Neurophysiology of the Vestibular System. 2nd ed. Philadelphia, PA: F. A. Davis, 1-301.

Baloh RW, Honrubia V. (2001) Bedside examination of the vestibular system. In: Clinical Neurophysiology of the Vestibular System. 3rd ed. New York, NY: Oxford University Press.
Baloh RW, Honrubia V, Jacobson K. (1987) Benign positional vertigo. Neurology 37:371-378.

Barber HO, Stockwell CW. (1980) Manual of Electronystagmography. 2nd ed. St. Louis, MO: Mosby.

Barber HO, Wright G. (1973) Positional nystagmus in normals. Adv Otorhinolaryngol 19:276-285.

Bertholon P, Bronstein AM, Davies RA, Rudge P, Thilo KV. (2002) Positional down beating nystagmus in 50 patients: cerebellar disorders and possible anterior semicircular canalithiases. J Neurol Neurosurg Psychiatry 72:366-372.

Bisdorff AR, Sancovic S, Debatisse D, Bentley C, Gresty MA, Bronstein AM. (2000) Positional nystagmus in the dark in normal subjects. Neuroophthalmology 24(1):283-290.

Bassani R, Torre DS. (2011) Positional nystagmus reversing from geotropic to apogeotropic: a new central vestibular syndrome. $J$ Neurol 258:313-315.

Brandt T. (1990) Positional and positioning vertigo and nystagmus. J Neurol Sci 95:3-28.

Cakir B, Ercan I, Cakir Z, Civelek S, Sayin I, Turgut S. (2006) What is the true incidence of horizontal semicircular canal benign paroxysmal positional vertigo? Otolaryngol Head Neck Surg 134: $451-454$.

Choi KD, Choi JH, Kim JS, Kim HJ, Kim MJ, Lee TH, Lee HL, Moon IS, Oh HJ, Kim JI. (2013) Rotational vertebral artery occlusion. Stroke 44(7):1817-1824.

Clarke AH, Ditterich J, Druen K, Schonfeld U, Steineke C. (2002) Using high frame-rate CMOS sensors for the three-dimensional eye tracking. Behav Res Methods Instrum Comput 34:549-560.

Clendaniel R, Landel R. (2007) Cervicogenic dizziness. In: Herdman S, ed. Vestibular Rehabilitation. 3rd ed. Philadelphia, PA: F. A. Davis.

Coats AC. (1993) Computer-quantified positional nystagmus in normals. Am J Audiol 14(5):314-326.

Davis RI, Mann RC. (1987) The effects of alerting tasks on caloric induced vestibular nystagmus. Ear Hear 8(1):58-60.

Eckert AM, Gizzi M. (1998) Video-oculography as part of the ENG battery. Br J Audiol 32:411-416.

Geisler C, Bergenious J, Brantberg K. (2000) Nystagmus findings in healthy subjects examined with infrared videonystagmoscopy. ORL J Otorhinolaryngol Relat Spec 62:266-269.

Glasauer S, Dieterich M, Brandt T. (2001) Central positional nystagmus simulated by a mathematical ocular mooto model of otolith-dependent modification of Listing's plane. J Neurophysiol 86:1546-1554.

Harrison MS, Ozsahinoglu C. (1972) Positional vertigo: aetiology and clinical significance. Brain 95:585-588.

Henry DF. (1999) Test-retest reliability of open-loop bithermal caloric irrigation responses from healthy young adults. Am J Otol 20(2):220-222.

Herishanu YO, Sharpe JA. (1981) Normal square wave jerks. Invest Ophthalmol Vis Sci 20(2):268-272.

Jacobson GP, Shephard NT, eds. (2008) Balance Function Assessment and Management. San Diego, CA: Plural Publishing Group. 
Jeffery H, Hopkins M, Anderson R, Patel V, Rogers J. (2017) The interpretation of static positional nystagmus in a balance clinic. Int $J$ Audiol 56:958-966.

Jongkees LBW. (1961) On Positional nystagmus. Acta Otolaryngol Suppl 159:78-83.

Jungmoo N, Seonhye K, Yongeun H, Ji Soo J. (2009) Ageotropic central positional nystagmus in nodular infarction. Neurology 73(14):1163.

Kitamura K, Ishida T, Kaminaga C, Haga M, Miyata M, Nomoto Y, Suzuki T. (1995) Infrared/video ENG recording of positional nystagmus in subjects with peripheral vestibular disease. Acta Otolaryngol Suppl 520:430-433.

Leigh RJ, Zee DS. (1999) The Neurology of Eye Movement. 3rd ed. New York, NY: Oxford University Press.

Lightfoot GR. (2004) The origin of order effects in the results of the bi-thermal caloric test. Int J Audiol 43(5):276-282.

Lin J, Elidan J, Baloh RW, Honrubia V. (1986) Direction-changing positional nystagmus: incidence and meaning. Am J Otolaryngol 7:306-310.

Maire R, Duvoisin B. (1999) Localization of static positional nystagmus with the ocular fixation test. Laryngoscope 109:606-611.

Margolis RH, Hunter LL. (1999) Typanometry: basic principles and clinical applications. In: Musiek FE, Rintelmann WI, eds. Contemporary Perspectives in Hearing Assessment. Boston, MA: Allyn and Bacon, 89-130.

Martens C, Goplen FK, Nordfalk KF, Aasen T, Glad Nordahl SH. (2016) Prevalence and characteristics of positional nystagmus in normal subjects. Otolaryngol Head Neck Surg 154(5):861-867.

McAuley JR, Dickman JD, Muatain W, Anand VK. (1996) Positional nystagmus in asymptomatic human subjects. Otolaryngol Head Neck Surg 114:545-553.

McGovern TN, Fitzgerald JE. (2008) The effect of mental alerting on peripheral vestibular nystagmus during spontaneous, gaze $\left(30^{\circ}\right.$ left, $30^{\circ}$ right) and body positional (left \& right lateral lying) testing using electronystagmography (ENG) Int $J$ Audiol 47(10): 601-606.

Mulch G, Lewitzki W. (1977) Spontaneous and positional nystagmus in healthy persons demonstrated only by electronystagmography: physiological spontaneous nystagmus or "functional scar"? Arch Ortorhinolaryngol 215:135-145.

Nam SJ, Kim SS, Huh SY, Kim SJ. (2009) Ageotropic central positional nystagmus in nodular infarction. Neurology 73(14):1163.
Nylén CO. (1950) Positional nystagmus. A review and future prospects. J Laryngol Otol 64:295-318.

Nylén CO. (1953) The posture test. Acta Otolaryngol Suppl 109: 125-130.

Roberts RA. (2016) Technique and interpretation of positional testing. In: Jacobson GP, Shepard NT, eds. Balance Function Assessment and Management. 2nd ed. San Diego, CA: Plural Publishing, 171-196.

Roberts RA, Gans RE. (2008a) Nonmedical management of positional vertigo. In: Jacobson GP, Shepard NT, eds. Balance Function Assessment and Management. San Diego, CA: Plural Publishing Group.

Roberts RA, Gans RE. (2008b) Background, technique, interpretation and usefulness of positional/positioning testing. In: Jacobson GP, Shepard NT, eds. Balance Function Assessment and Management. San Diego, CA: Plural Publishing, 171-196.

Rogers J, Anderson R, Patel C. (2014) Establish new normal reference ranges for nystagmus slow-phase velocity (SPV) using videonystagmography (VNG) for gaze, body positional and head shake testing. BAAA Conference 2014. Bournemouth International Center, UK, 2014. November 20-21. Poster Presentation.

Rubin W, Brookler KH. (1991) Dizziness: Etiologic Approach to Management. New York, NY: Thieme Medical Publishers.

Shepard N, Telian S. (1996) Practical Management of the Balance Disorder Patient. San Diego, CA: Singular Publishing Group.

Sunami K, Tochino R, Zushi T, Yamamoto H, Tokuhara Y, Iguchi H, Takayama M, Konishi K, Yamane H. (2004) Positional and positioning nystagmus in healthy subjects under videonystagmoscopy. Acta Otolaryngol Suppl 554:35-37.

Uemura T, Suzuki JI, Hozawa J. (1977) Neuro-Otological Examination with Special Reference to Equilibrium Function Tests. Baltimore, MD: University Park.

Vitte E, Semont A. (1995) Assessment of vestibular function by videonystagmography. J Vestib Res 5:377-383.

Waldorf RA, Haenel J, Kohut RI, Kerle JK. (1977) The observation, using an infrared video system, of patients' eye movements resulting from vestibular stimulations and their comparisons with electronystagmographic data. Trans Sect Otolaryngol Am Acad Ophthalmol Otolaryngol 84(2):392-399.

Watson P, Barber HO, Deck J, Terbrugge K. (1981) Positional vertigo and nystagmus of central origin. Can J Neurol Sci 8:133-137. 
APPENDIX: Clinical flowchart for static positional testing.

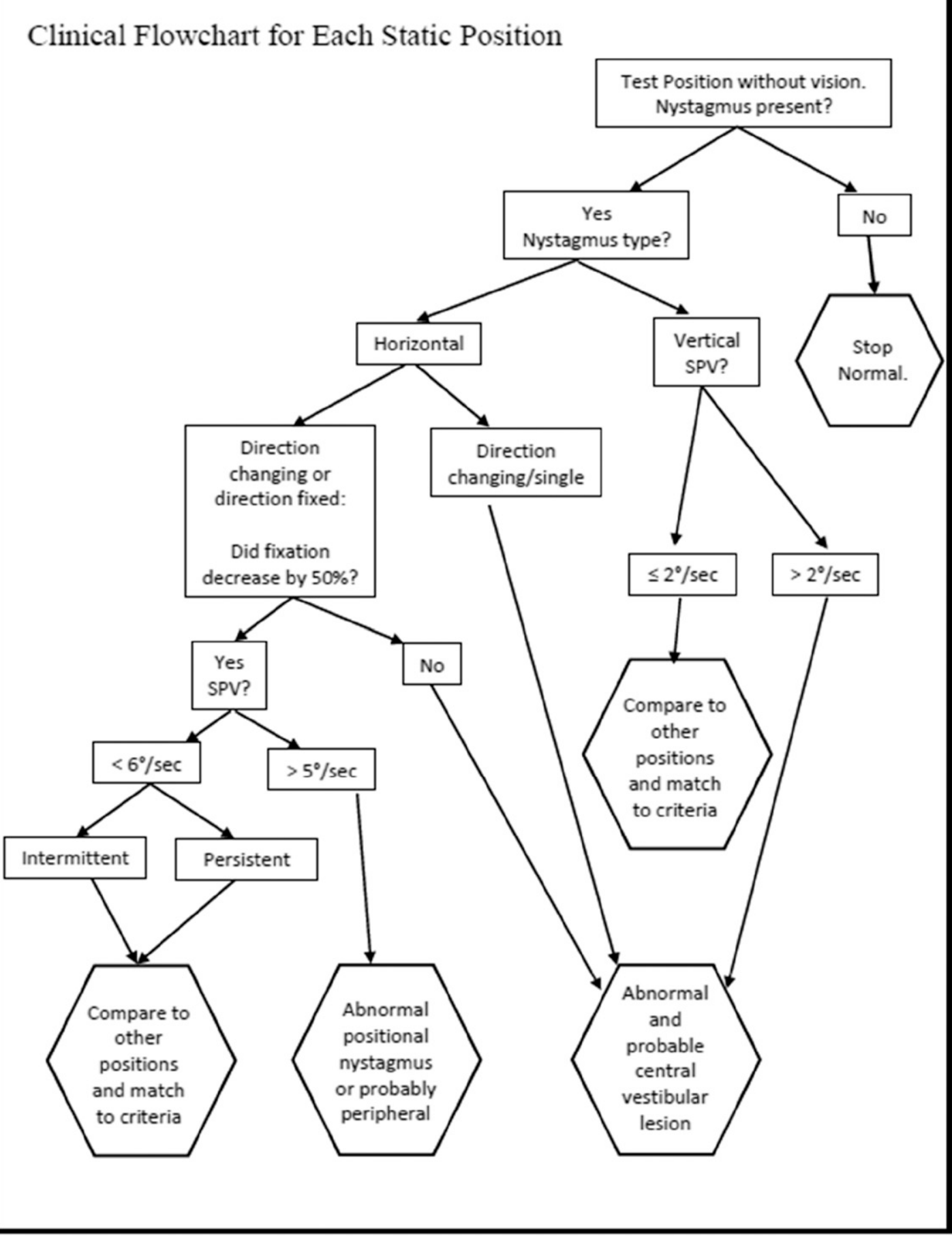

